\title{
Evidence of Germline Mosaicism for a Novel BCOR Mutation in Two Indian Sisters with Oculo-Facio-Cardio-Dental Syndrome
}

\author{
Sumita Danda ${ }^{a}$ Vanessa A. van Rahden ${ }^{d}$ Deepa John ${ }^{b}$ Padma Paul $^{b}$ \\ Renu Raju ${ }^{b}$ Santosh Koshy ${ }^{c}$ Kerstin Kutsche ${ }^{d}$ \\ Departments of a Clinical Genetics, ${ }^{b}$ Ophthalmology and ${ }^{\mathrm{c}}$ Dental Surgery, Christian Medical College and Hospital, \\ Vellore, India; ${ }^{\mathrm{d}}$ Institute of Human Genetics, University Medical Center Hamburg-Eppendorf, Hamburg, Germany
}

\section{Key Words}

BCL6 corepressor gene $\cdot$ Gonadal mosaicism $\cdot$ India .

Microphthalmia $\cdot$ Radiculomegaly $\cdot$ X-linked

\begin{abstract}
In this study, we report on 2 sisters from India with oculofacio-cardio-dental (OFCD) syndrome caused by a novel heterozygous mutation c.3490C $>T$ (p.R1164*) in the BCOR gene. OFCD syndrome is an X-linked inherited disorder which is lethal in males. Interestingly, both parents of the sisters were phenotypically normal, and DNA analysis from blood and buccal or saliva cells failed to detect the BCOR mutation found in their 2 daughters. To the best of our knowledge, for the first time, we provide indirect evidence of germline mosaicism for the BCOR mutation in one of the parents of the 2 sisters affected by OFCD syndrome. Although this condition is lethal in males, gonadal mosaicism could also be present in the father. The relevance of clinical diagnosis and mutation analysis required for genetic counseling is described in this family.

(c) 2014 S. Karger AG, Basel
\end{abstract}

Oculo-facio-cardio-dental (OFCD) syndrome (MIM 300166) is a rare $\mathrm{X}$-linked male-lethal condition manifesting in females. It comprises characteristic eye, facial, cardiac, and dental abnormalities [Hilton et al., 2009], with only 1 report on an Indian family so far [Rudrappa et al., 2010]. Heterozygous loss-of-function mutations in the $B C O R$ gene in Xp11.4 have been identified in individuals with OFCD [Ng et al., 2004] and include submicroscopic deletions as well as sequence-level alterations such as missense, nonsense, splice-site, and frameshift mutations $[\mathrm{Ng}$ et al., 2004; Horn et al., 2005; Oberoi et al., 2005; Hilton et al., 2009; Jiang et al., 2009; Davoody et al., 2011; Kondo et al., 2012; Lozic et al., 2012; Feberwee et al., 2014]. Usually, mutations are transmitted from an affected mother to her daughter(s) or occur de novo in girls with OFCD syndrome [Hedera and Gorski, 2003; Horn et al., 2005; McGovern et al., 2006; Rudrappa et al., 2010; Kondo et al., 2012; Lozic et al., 2012; Feberwee et al., 2014]. Germline mosaicism has not been reported up to date.

Here, molecular analysis of 2 sisters from India with clinical diagnosis of OFCD syndrome revealed a novel

\section{KARGER}

E-Mail karger@karger.com

www.karger.com/msy (c) 2014 S. Karger AG, Basel

$1661-8769 / 14 / 0055-0251 \$ 39.50 / 0$
Prof. Sumita Danda

Department of Clinical Genetics

Christian Medical College and Hospital

Vellore, Tamil Nadu 632004 (India)

E-Mailsdanda@cmcvellore.ac.in 
mutation in the $B C O R$ gene leading to a premature stop codon. We describe the clinical phenotype and molecular results of the whole family including the parents, the importance of genetic counseling and provide a review of the literature.

\section{Case Reports}

The proband, an 8-year-old girl, and her 6-year-old sister were referred to the Clinical Genetics out-patient unit of the Christian Medical College, Vellore by the Ophthalmology Department for syndrome diagnosis. The proband was born after an uneventful pregnancy by an uncomplicated vaginal delivery (birth weight 3.75 $\mathrm{kg}$ ). Her parents are non-consanguineous. At birth, bilateral cataract was noted which was operated. At the age of 8 months, she underwent another surgery as she had been diagnosed with double outlet right ventricle, ventricular septal defect and pulmonary stenosis. Simultaneously, an anoplasty for a rectovaginal fistula was performed. Another intracardiac repair at the age of 3 was performed. She had delayed dentition; speech developed at 2.5 years, and she was able to walk at 3 years of age.

On physical examination of the proband, short stature was observed $(122 \mathrm{~cm} ;<3 \mathrm{SD})$. Weight and head circumference were normal. She had facial dysmorphism including high forehead, frontal bossing, broad nasal tip with bifid cartilage, low set, posteriorly rotated ears, small ear lobes, and microcornea. Intraoral examina- tion showed delayed eruption of permanent dentition, class I malocclusion with deep overbite, V-shaped maxilla with high-arched palatal vault and bifid uvula (fig. 1a-d). Camptodactyly of the 4th and 5th fingers (right $>$ left), proximally placed thumbs, restricted supination and pronation of the left forearm, camptodactyly and syndactyly of 2 nd and 3 rd toes, and sandal gap were present (fig. 1e, f). She had nystagmus and scars on the chest and abdomen from previous surgeries. The rest of the examination was normal. The panoramic radiographic findings of the proband showed radiculomegaly in relation to lower incisors with open apices, delayed eruption of permanent teeth and delayed shedding of deciduous upper anterior teeth (fig. 2a). The maxillary and mandibular canines are still in formation stage. The lateral cephalometric examination revealed skeletal class I with severe vertical growth pattern, increased gonial angle, steep mandibular plane with retroclined incisors, and competent lips (fig. 2b). Elbow radiographs at infancy showed left radioulnar synostosis (fig. 2c).

Her sister had congenital cataract that was operated 20 days after birth. Her milestones were normal, and she did not undergo any other surgeries. On examination, she had short stature $(111 \mathrm{~cm},<3$ $\mathrm{SD}$ ), frontal bossing, microcornea, high nasal bridge, $\mathrm{V}$-shaped maxilla with high palate, and sandal gap between the 1st and 2nd toes (fig. 3, left panel). Overall milder skeletal anomalies were noted in the sister. The panoramic radiographs showed delayed eruption of first molars and presence of all permanent tooth buds. However, radiculomegaly could not be diagnosed due to the younger age of the patient. The lateral cephalometric radiograph showed skeletal class II attributable to retrognathic mandible which is a normal finding re-
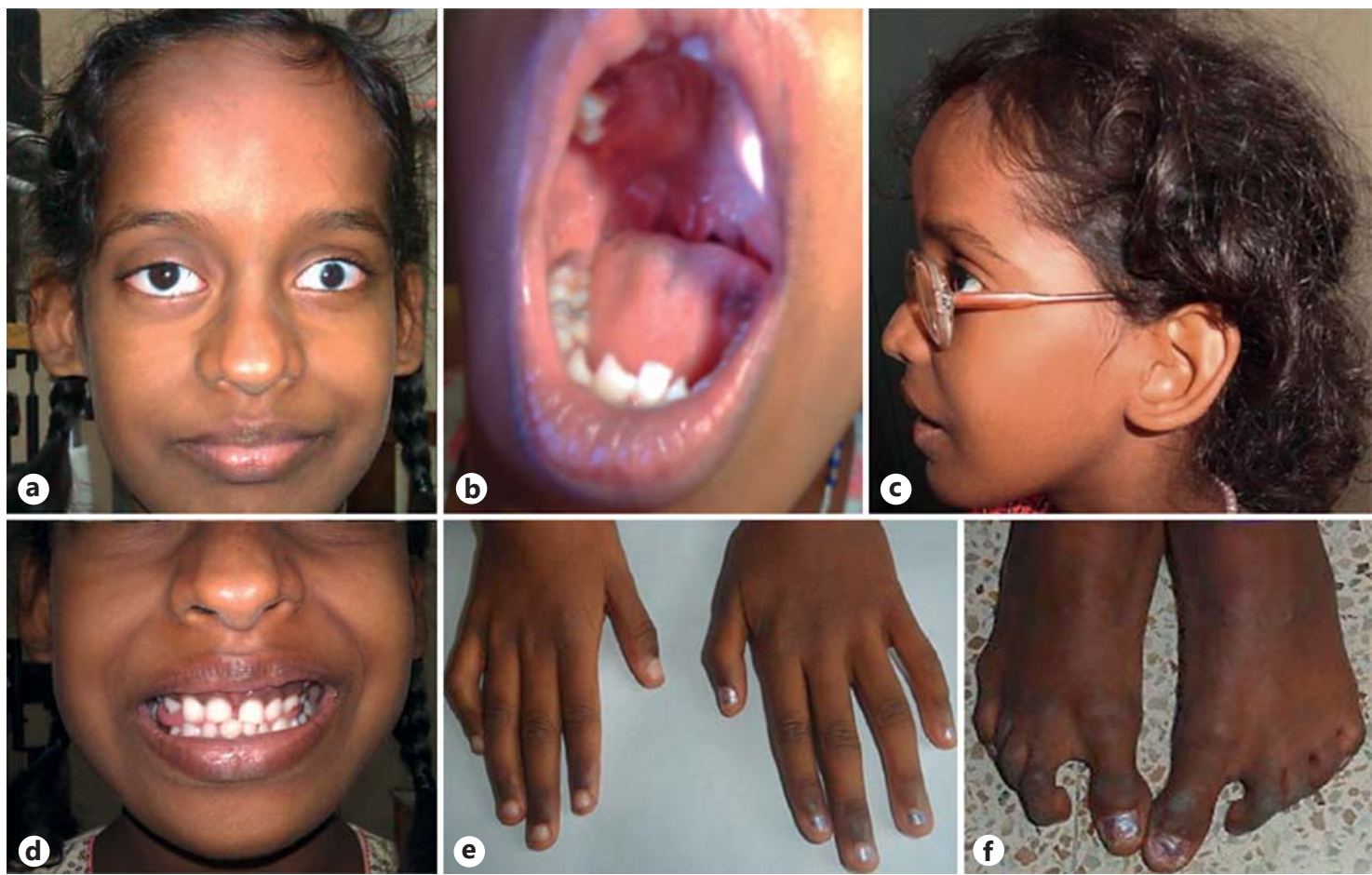

Fig. 1. Photographs of the proband with OFCD. a-d The front profile, bifid uvula, side profile, and malocclusion of teeth are shown. e Camptodactyly of 4 th and 5th fingers. f Feet showing sandal gaps, syndactyly and camptodactyly of toes. 
garding the age of the patient. Other features were vertical growth pattern, increased gonial angle, steep mandibular plane, retroclined upper incisors, and competent lips (fig. 3, right panel).

The parents were healthy, had normal height and did not have any dysmorphic features apart from sandal gaps.

Although an initial diagnosis of VACTERL had been made elsewhere, our evaluation made the clinical diagnosis of OFCD syndrome more likely. Routine chromosome analysis revealed a normal karyotype in both sisters. Some cases with Fanconi anemia can also present as VACTERL; cell cultures treated with mitomycin C (done earlier) revealed that the proband had increased chromosomal breakage, which was not the case in her sister's blood.

Based on the clinical diagnosis OFCD syndrome, the proband and her sister underwent molecular analysis of the BCOR gene; the parents were tested for the mutation in blood and saliva or buccal cell-derived DNA.

\section{Material and Methods}

\section{Patients}

We obtained clinical data and blood samples from the 2 affected sisters and their parents. In addition, buccal swabs and saliva were received from the mother and the father, respectively. Clinical data and samples were obtained with informed consent, including consent to use the photographs in this report, under protocols approved by the Institutional Review Board.
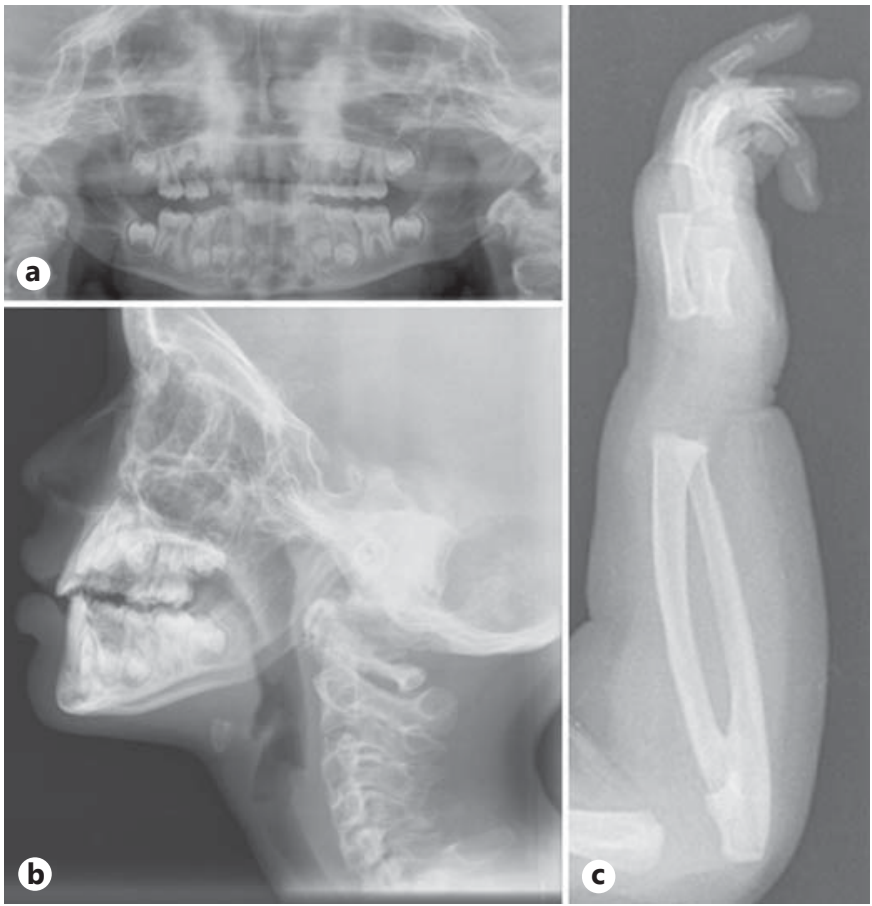

b

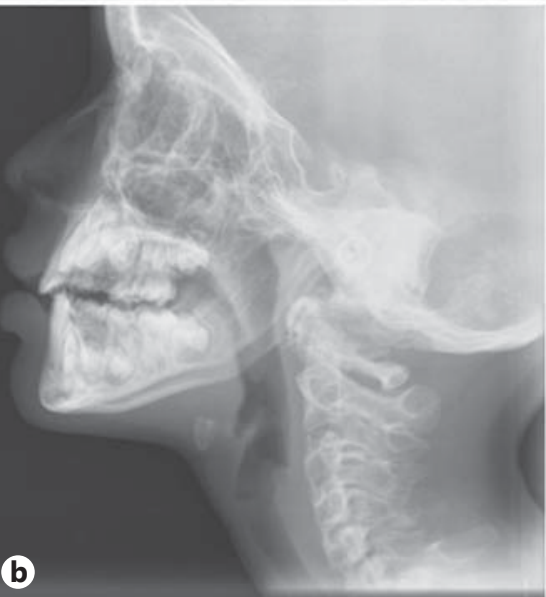

Fig. 2. a Orthopantomogram showing radiculomegaly with open apices. b Lateral cephalometric radiograph. c Lateral left elbow radiograph (at infancy) showing radioulnar synostosis.
Fig. 3. Left panel: facial profile and feet of the proband's sister. Right panel: orthopantomogram and cephalometric radiograph.
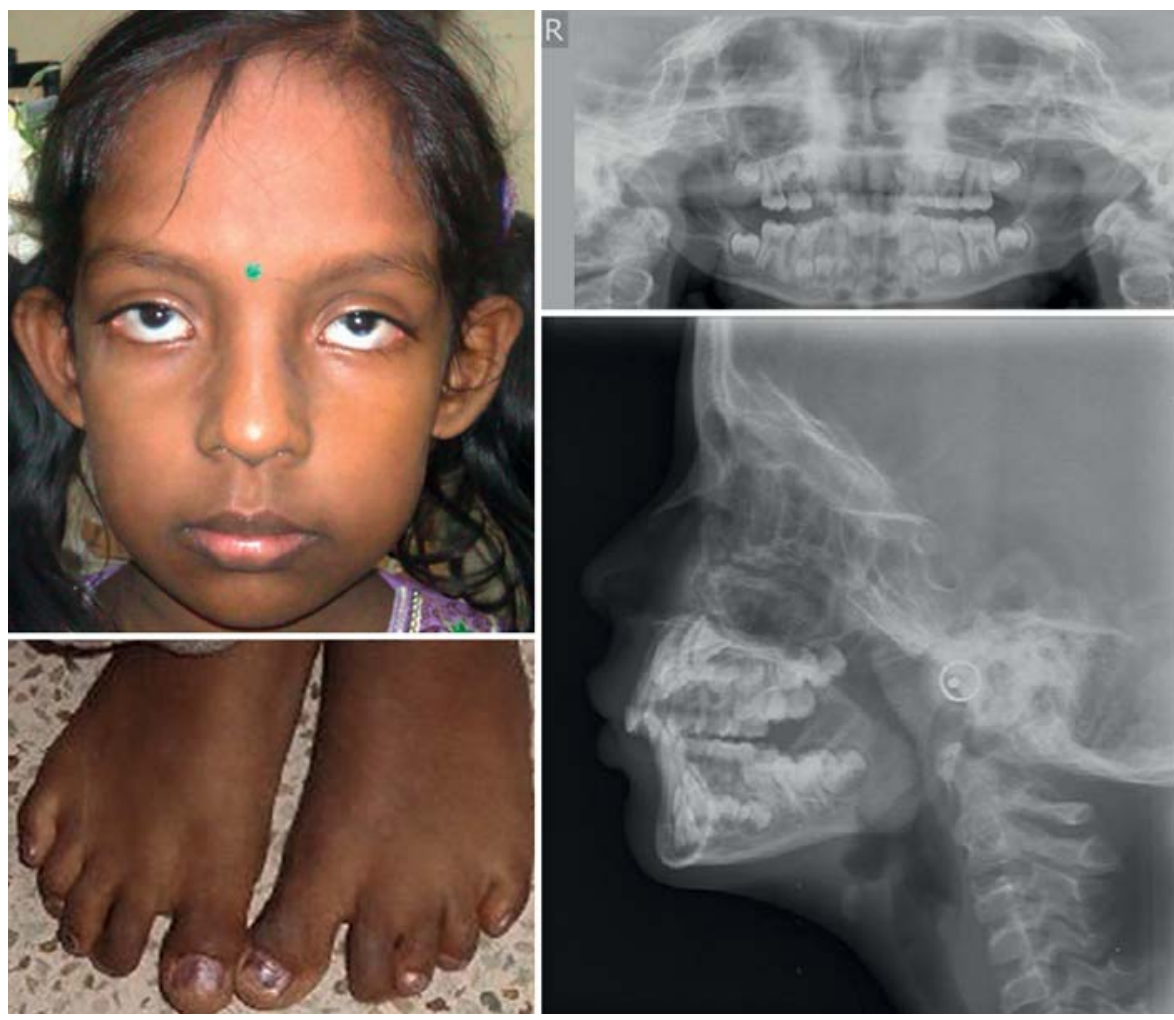
Sequencing of $B C O R$

We isolated genomic DNA from blood, saliva samples and buccal swabs by standard procedures. The coding exons $4,6,7$, and 12 of the BCOR gene (GenBank accession no. NM_001123385) were amplified from genomic DNA of the proband, while only exon 7 was amplified from DNA samples of the proband's sister and parents. Primer sequences are available on request. We directly sequenced amplicons using the ABI Big Dye Terminator Sequencing Kit (Applied Biosystems, Weiterstadt, Germany) and an automated capillary sequencer (ABI 3500; Applied Biosystems). Sequence electropherograms were analyzed using the Sequence Pilot software (JSI Medical Systems, Kippenheim, Germany). Declared relationships were verified by genotyping both parents and their daughters at 6 microsatellite loci.

\section{Results}

Sequencing of selected exons of the BCOR gene from leukocyte-derived DNA of the proband revealed the heterozygous nonsense mutation c.3490C $>\mathrm{T}$ (p.R1164*) in exon 7 that has not yet been described (fig. 4). This transition leads to the introduction of a premature stop codon and is a loss-of-function mutation, likely representing the genetic cause of OFCD syndrome in the proband. In the affected sister, sequencing of $B C O R$ exon 7 revealed the same heterozygous mutation (data not shown). However, we could not detect the c.3490C $>$ T substitution in genomic DNA isolated from leukocytes (fig. 4), buccal or saliva cells from the 2 asymptomatic parents (data not shown).

\section{Discussion}

In this study, we describe 2 sisters affected by OFCD syndrome. Both presented with congenital cataracts operated shortly after birth. The proband was more severely affected with cardiac, skeletal and dental anomalies and significant facial dysmorphism than her sibling who did not have any heart problems.

OFCD syndrome is caused by germline mutations in the BCOR gene. BCOR encodes the BCL6 corepressor, a ubiquitously expressed nuclear protein which was first identified as a corepressor that interacts with the transcriptional repressor BCL6 through the BCORBCL-6 binding domain (BCOR ${ }^{\mathrm{BBD}}$ ) [Huynh et al., 2000]. The target genes of BCOR are TP53 and Cyclin D2 (CCND2) in B cells [Gearhart et al., 2006]. BCOR is a key transcriptional regulator during embryogenesis [Ng et al., 2004] and plays a role in differentiation of multiple tissue lineages during early embryonic devel-

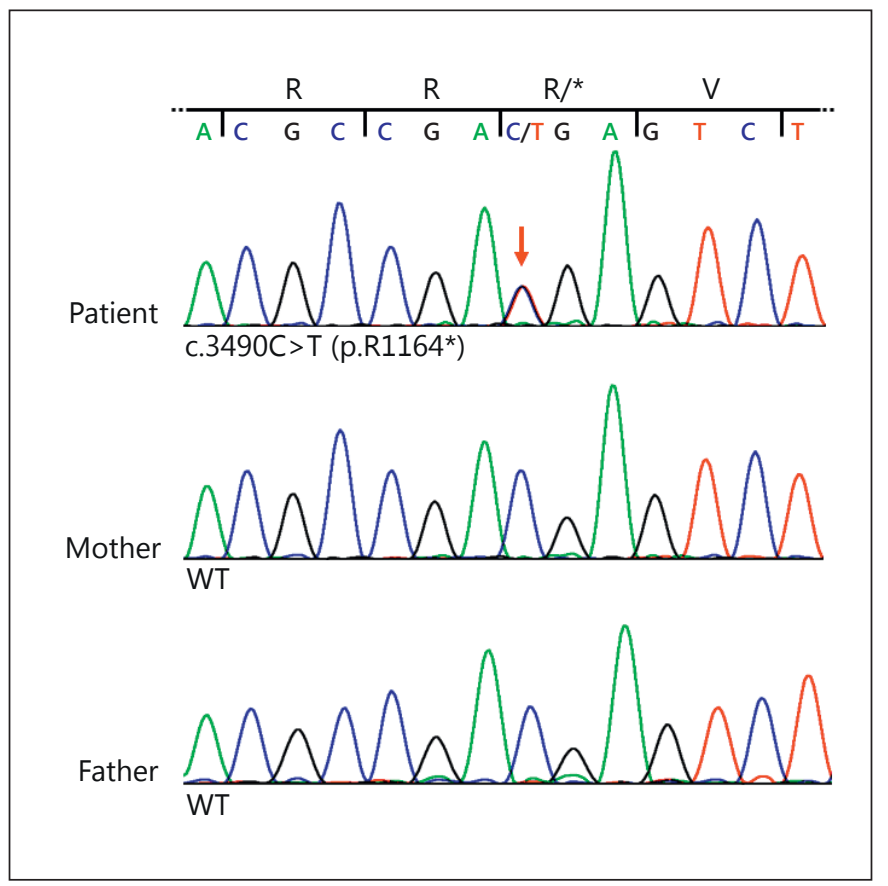

Fig. 4. $B C O R$ mutation in the 2 sisters with OFCD syndrome. The $B C O R$ nonsense mutation c. $3490 \mathrm{C}>\mathrm{T}$ (p.R1164*) is present in the 2 sisters but not in their parents. Sequence electropherograms from leukocyte-derived genomic DNA of the proband (upper panel), her mother (middle panel) and her father (lower panel). The index patient is heterozygous for the nonsense mutation c.3490C>T (p. R1164*) in exon 7 of BCOR, while the parents show wild-type (WT) sequence. Nucleotide triplets and encoded amino acids are indicated.

opment [Wamstad et al., 2008]. It has also been identified as a negative regulator of osteo-dentiogenic capacity of mesenchymal stem cells [Fan et al., 2009]. More recent data indicate a cooperative function of the zebrafish orthologs Bcor and Bcl6a in normal optic cup formation through regulating p53-dependent apoptosis [Lee et al., 2013].

Both familial and sporadic cases with OFCD syndrome have been described in the literature, where in familial cases both mother and daughter(s) were affected [Hedera and Gorski, 2003; McGovern et al., 2006; Kondo et al., 2012; Lozic et al., 2012]. The 2 sisters with OFCD from India described here have a novel alteration in $B C O R$, the heterozygous nonsense mutation c. $3490 \mathrm{C}>\mathrm{T}$ (p.R1164*). Interestingly, none of the parents showed any clinical expression of the disease. Though clinical features were absent, we performed DNA analysis from 2 different tissues (blood and buc$\mathrm{cal} /$ saliva cells) of both parents to identify possible somatic mosaicism. The BCOR mutation c.3490C $>\mathrm{T}$ 
could not be detected in the various DNA samples analyzed from the parents suggesting that either the mother or the father is carrier of gonadal cell mosaicism. Germline mosaicism has been reported in other Xlinked disorders, such as $D C X$-related disorders, bilateral periventricular nodular heterotopia, Rett syndrome, and Goltz-Gorlin syndrome [Mari et al., 2005; Hehr et al., 2007; Maas et al., 2009; Kasper et al., 2013]. This has important implications for genetic counseling. Gonadal mosaicism is a rare phenomenon reported in several autosomal dominant, X-linked and, exceptionally, in autosomal recessive disorders [Saranjam et al., 2013]. Though parents are phenotypically normal in such cases, and a mutation is not detectable in blood, the recurrence risk to couples will be $\leq 50 \%$, as shown for other conditions [Zlotogora, 1998]. The exact figure depends on the proportion of affected cells in the gonads, an estimation of which is difficult to give. With the present study, OFCD is added to the list of disorders in which gonadal mosaicism can occur. Prenatal diagnosis should be offered in cases of OFCD, irrespective of parental phenotype or molecular analysis status in simplex as well as multiplex cases.

Recurrent clonal and somatic mutations in $B C O R$ have recently been detected in some cases with acute myelogenous leukemia who were subjected to whole exome sequencing [Grossmann et al., 2011]. Looking retrospectively, an increased mitomycin C-induced chromosome breakage in the proband of our family supports a role of $\mathrm{BCOR}$ in one of the pathways for leukemogenesis. In the study of Grossmann et al. [2011], somatic BCOR mutations were of disruptive nature and constantly targeted the only functional allele in both male and female patients and denoted a loss-of-function of the tumor suppressor gene. Patients with Fanconi anemia often develop AML or myelodysplastic syndrome in their teens or young adulthood due to a strong selective pressure in the bone marrow that leads to clonal evolution [Soulier, 2011]. $B C O R$ mutations are therefore potentially implicated as one of the possible causes of AML developing in Fanconi anemia. Pancreatic endocrine microadenomatosis has been reported in a single case of OFCD [Atiq et al., 2012], and no other malignancies are reported in the literature. The last hematological profile of the proband, at the time the manuscript was being revised, was normal. Till further guidelines on management of OFCD are available, a yearly follow-up of the proband will be done to check growth and any abnormal hematological parameters. The parents were counseled accordingly and also offered prenatal diagnosis for potential future pregnancies. Further studies are required to evaluate which pathways are affected by disruption of the BCOR gene. Though there are no reported cases of hematological cancers in OFCD, further research in this area is warranted.

\section{Acknowledgement}

We are grateful to the patients and their family who contributed to this study. This work was supported by a grant from the Deutsche Forschungsgemeinschaft (KU 1240/6-1 to K.K.).

\section{References}

Atiq M, Gong Y, Raju GS, Lee JH: Pancreatic endocrine microadenomatosis in a patient with oculofaciocardiodental (OFCD) syndrome. Pancreas 41:327-329 (2012)

Davoody A, Chen IP, Nanda R, Uribe F, Reichenberger EJ: Oculofaciocardiodental syndrome: a rare case and review of the literature. Cleft Palate Craniofac J 49:e55-e60 (2011).

- Fan Z, Yamaza T, Lee JS, Yu J, Wang S, et al: $\mathrm{BCOR}$ regulates mesenchymal stem cell function by epigenetic mechanisms. Nat Cell Biol 11:1002-1009 (2009)

-Feberwee H, Feenstra I, Oberoi S, Sama I, Ockeloen $\mathrm{C}$, et al: Novel $B C O R$ mutations in patients with oculofaciocardiodental (OFCD) syndrome. Clin Genet 85:194-197 (2014).

- Gearhart MD, Corcoran CM, Wamstad JA, Bardwell VJ: Polycomb group and SCF ubiquitin ligases are found in a novel BCOR complex that is recruited to BCL6 targets. Mol Cell Biol 26:6880-6889 (2006).
Grossmann V, Tiacci E, Holmes AB, Kohlmann A, Martelli MP, et al: Whole-exome sequencing identifies somatic mutations of $B C O R$ in acute myeloid leukemia with normal karyotype. Blood 118:6153-6163 (2011).

Hedera P, Gorski JL: Oculo-facio-cardio-dental syndrome: skewed $\mathrm{X}$ chromosome inactivation in mother and daughter suggest X-linked dominant inheritance. Am J Med Genet A 123A:261-266 (2003).

Hehr U, Uyanik G, Aigner L, Couillard-Despres S, Winkler J: DCX-related disorders, in Pagon RA, Bird TD, Dolan CR, Fong CT, Stephens K (eds): GeneReviews [internet] (University of Washington, Seattle 2007).

Hilton E, Johnston J, Whalen S, Okamoto N, Hatsukawa Y, et al: $B C O R$ analysis in patients with OFCD and Lenz microphthalmia syndromes, mental retardation with ocular anomalies, and cardiac laterality defects. Eur J Hum Genet 17:1325-1335 (2009).
Horn D, Chyrek M, Kleier S, Lüttgen S, Bolz H, et al: Novel mutations in BCOR in three patients with oculo-facio-cardio-dental syndrome, but none in Lenz microphthalmia syndrome. Eur J Hum Genet 13:563-569 (2005).

Huynh KD, Fischle W, Verdin E, Bardwell VJ: BCoR, a novel corepressor involved in BCL-6 repression. Genes Dev 14:1810-1823 (2000).

- Jiang YH, Fang P, Adesina AM, Furman P, Johnston JJ, et al: Molecular characterization of cooccurring Duchenne muscular dystrophy and X-linked oculo-facio-cardio-dental syndrome in a girl. Am J Med Genet A 149A:12491252 (2009).

- Kasper BS, Kurzbuch K, Chang BS, Pauli E, Hamer HM, et al: Paternal inheritance of classic X-linked bilateral periventricular nodular heterotopia. Am J Med Genet A 161A:13231328 (2013).
Germline Mosaicism in Oculo-Facio-

Cardio-Dental Syndrome
Mol Syndromol 2014;5:251-256 DOI: $10.1159 / 000365768$ 
Kondo Y, Saitsu H, Miyamoto T, Nishiyama K, Tsurusaki Y, et al: A family of oculofaciocardiodental syndrome (OFCD) with a novel BCOR mutation and genomic rearrangements involving NHS. J Hum Genet 57:197-201 (2012).

Lee J, Lee BK, Gross JM: Bcl6a function is required during optic cup formation to prevent p53-dependent apoptosis and colobomata. Hum Mol Genet 22:3568-3582 (2013).

Lozic B, Ljubkovic J, Panduric DG, Saltvig I, Kutsche K, et al: Oculo-facio-cardio-dental syndrome in three succeeding generations: genotypic data and phenotypic features. Braz J Med Biol Res 45:1315-1319 (2012).

-Maas SM, Lombardi MP, van Essen AJ, Wakeling EL, Castle B, et al: Phenotype and genotype in 17 patients with Goltz-Gorlin syndrome. J Med Genet 46:716-720 (2009).
- Mari F, Caselli R, Russo S, Cogliati F, Ariani F, et al: Germline mosaicism in Rett syndrome identified by prenatal diagnosis. Clin Genet 67:258-260 (2005)

McGovern E, Al-Mudaffer M, McMahon C, Brosnahan D, Fleming P, Reardon W: Oculo-facio-cardio-dental syndrome in a mother and daughter. Int J Oral Maxillofac Surg 35:10601062 (2006).

Ng D, Thakker N, Corcoran CM, Donnai D, Perveen $\mathrm{R}$, et al: Oculofaciocardiodental and Lenz microphthalmia syndromes result from distinct classes of mutations in BCOR. Nat Genet 36:411-416 (2004).

- Oberoi S, Winder AE, Johnston J, Vargervik K, Slavotinek AM: Case reports of oculofaciocardiodental syndrome with unusual dental findings. Am J Med Genet A 136:275-277 (2005).
Rudrappa S, Kumar R, Kumar GS: Oculo-facio-cardio-dental syndrome in a girl and her mother. Indian J Hum Genet 16:169-171 (2010).

- Saranjam H, Chopra SS, Levy H, Stubblefield BK, Maniwang E, et al: A germline or de novo mutation in two families with Gaucher disease: implications for recessive disorders. Eur J Hum Genet 21:115-117 (2013).

- Soulier J: Fanconi anemia. Hematology Am Soc Hematol Educ Program 2011:492-497 (2011).

Wamstad JA, Corcoran CM, Keating AM, Bardwell VJ: Role of the transcriptional corepressor Bcor in embryonic stem cell differentiation and early embryonic development. PLoS One 3:e2814 (2008)

Zlotogora J: Germ line mosaicism. Hum Genet 102:381-386 (1998). 